\title{
Modelling and Pricing of Catastrophe Risk Bonds with a Temperature-Based Agricultural Application
}

\author{
N. KARAGIANNIS $\dagger$, H. ASSA $\dagger^{*}$, A. A. PANTELOUS $\dagger$ and C. G. TURVEY $\ddagger$ \\ $\dagger$ Department of Mathematical Sciences and Institute for Risk and Uncertainty, University of \\ Liverpool, Peach Street, L697ZL, Liverpool United Kingdom \\ $\ddagger$ Charles H. Dyson School of Applied Economic and Management, Cornell University, 450A \\ Warren Hall, Ithaca, NY 14853 USA
}

(Received: 13 December 2015)

\begin{abstract}
Catastrophe risk bonds are always within a multi-asset class portfolio of alternative risk premia in many hedge funds. In this paper, we consider an over the counter (OTC) insurance contract on catastrophe risk between an insurance company and a hedge-fund. The contract acts as a bond within which the insurance company, which issues the bond, pays payments higher than the market risk free interest, in order to be insured against the risk of a predefined natural catastrophe. The contract is priced by the utility indifference pricing method. We apply our framework to price agricultural catastrophe (AgriCAT) bonds in two cities in Iran where their harvests are exposed to the risk of low temperature.
\end{abstract}

Keywords: Catastrophe Risk Bonds; Insurance; Hedge Fund; Over The Counter (OTC); Utility Indifference Pricing Method; Agricultural Catastrophes

JEL Classification: G22; C61; C63; G12

\section{Introduction}

\subsection{Motivation}

In the last three decades, there is a consistently increasing growth of insured losses due to nature related catastrophic events (i.e. sudden events in time that cause significant losses to one person or a group of people) which has pressured the reinsurance industry to consider and develop alternative risk transfer products. These products are designed to alleviate (the whole or) part of their risks by implementing securitization mechanisms to achieve access to adequate liquidity funds. Among them, Catastrophe (CAT) risk bonds, designed to transfer the financial consequences of catastrophic events (such as floods, hurricanes or earthquakes) from the issuers to investors have become popular. CAT risk bonds have been shown to successfully cover the insureds' liabilities while protecting also traditional reinsurance providers and the governmental budgets. The Insurance Service Office's (ISO's) Property Claim Service $(\mathrm{PCS})^{1}$ declared 254 catastrophes that incurred damages of approximately US $\$ 112$ billion between 1990 and 1996, while only the losses

\footnotetext{
*Corresponding author. Email: assa@liverpool.ac.uk

${ }^{1}$ ISO's Property Claim Service unit is an internationally recognized authority in the USA, Puerto Rico and the US Virgin Islands on insured property losses due to catastrophes and contains information on all the historical catastrophes occurred in these areas since 1949, including the states affected, perils, and associated loss estimates. (http://www.verisk.com/ property-claim-services/).
} 
due to Hurricane Andrew in 1992 reached US\$ 26 billion ${ }^{2}$. Thus, even a single event can jeopardize the insurance companies solvency.

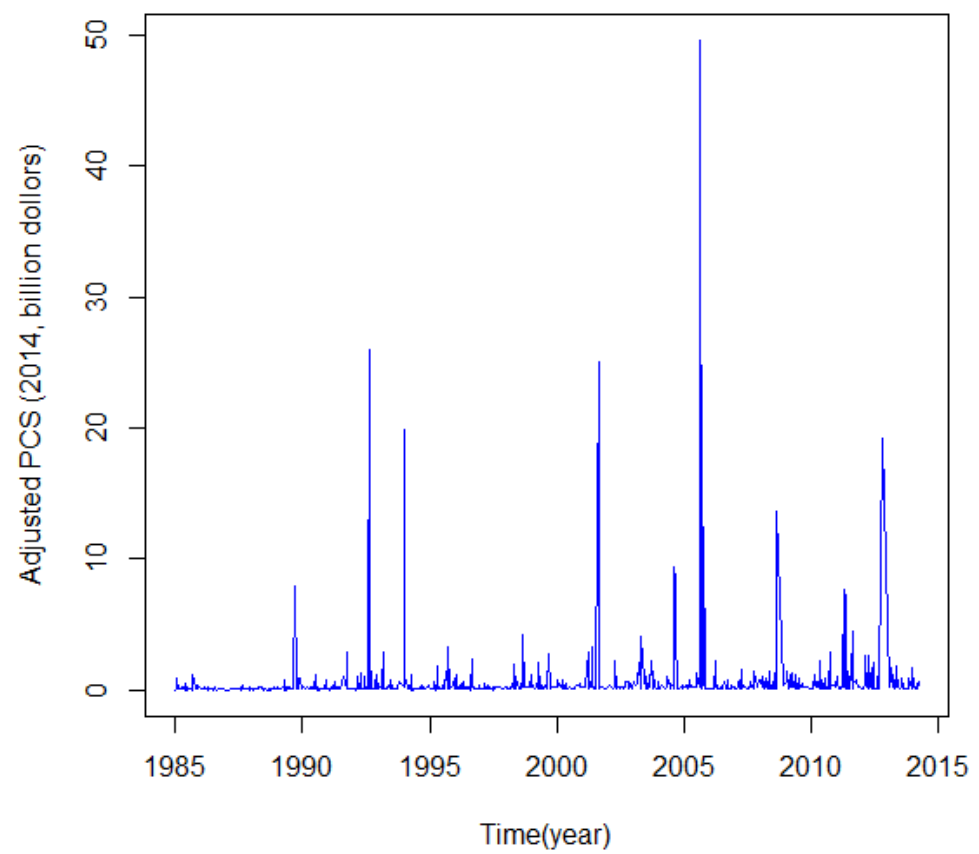

Figure 1. Annual catastrophe losses in millions US\$ in the USA during 1985-2013, data from PCS; see Shao et al. (2015b). Particularly, we can distinguish the catastrophe related losses from the Northridge earthquake (1994) with total insured cost of US $\$ 20$ billion, the Hurricane Katrina (2005) with total insured cost of US $\$ 45$ billion and the Hurricane Sandy (2012) with total insured cost of US\$20 billion.

As the insured risks are transferred through CAT risk bonds in the financial markets, investors accept to buy a particular set of risks related to predetermined catastrophic events attractive risk premium. However, if some (or all) of the predefined catastrophic events occur, the investors can lose part or all of the principal invested and the issuers (often insurance or reinsurance companies) receive that money to cover insured losses.

Given the significant up-tick in catastrophic events, and a significant demand in the insurance and reinsurance industries there is a great interest in designing, developing and pricing CAT risk bonds. This is true of weather related agricultural risks - the main area of this paper - as well as property-casualty. Analytically, in this paper, we provide a pricing method for CAT risk bonds using a utility indifference pricing methodology. As an interesting application a specific CAT risk bond related to agricultural catastrophes is designed analytically and priced. Thus, the Agricultural CAT (AgriCAT) risk bond transfers the risk of a catastrophe to a hedge-fund. The underlying bond is assumed to be issued in distinct tranches with different payoffs, which occur with differing probability. Each of these tranches is considered as a zero coupon type bond.

\footnotetext{
${ }^{2}$ An illustration of the PCS catastrophe loss data converted to 2014 US\$ using the Consumer Prices Index (CPI) in US is given in Figure 1.
} 


\subsection{The Market for Catastrophe Risk Bonds}

While the previous section made the economic case for focusing on agricultural risks, this section provides a general overview of CAT risk bonds in general. Losses and recovery costs from the occurrence of catastrophic events are covered typically by an appropriate portfolio of utility companies, special insurance programs and/or governmental budgets. The requirement to achieve adequate liability coverage is to have a system that has adequate financial depth to fulfill claims. To succeed, financing is essential using special purpose instruments from the global financial market.

CAT risk bonds are inherently risky, non-indemnity-based multi-period deals, which pay a regular coupon to investors at the end of each period and a final principal payment at the maturity date, if no predetermined catastrophic events occur. A major catastrophe in the secured region before the CAT risk bond maturity date leads to full or partial loss of the capital. CAT risk bonds, are designed to transfer the liability risk triggered by a catastrophic event from the insured to the global markets. This financial instrument can be a significant source of cash flow, large enough to underwrite big losses. CAT risk bonds have become very popular insurance-linked financial securities over the last decades and their use has been accelerating.

The first experimental transaction was completed in the mid-1990s after Hurricane Andrew and the Northridge earthquake, which incurred insurance losses of billions od dollars, respectively, by a number of specialized catastrophe-oriented insurance and reinsurance companies in the USA, including AIG, Hannover Re, St Paul Re, and USAA (see GAO (2002)). The issued capital increased tenfold within the ten years, from less than US $\$ 0.8$ billion in 1997 to over US $\$ 8$ billion in 2007. The issuers raised more than US $\$ 9$ billion of new CAT bonds in $2014^{3}$.

Moreover, it should be mentioned because CAT risk bonds are (at least ex-ante) uncorrelated with the traditional stock markets CAT risk bond investors can also gains in bad economic circumstances.

Practically speaking, in order to bear the catastrophe risks, CAT risk bonds carry a 1 to 5years maturity and compensate for a floating LIBOR coupon plus a premium at a rate between $2 \%$ and $20 \%$ (see Cummins (2008) and GAO (2002)). One of the key elements of any CAT risk bond is the terms under which the securities begin to experience a loss. Their payoff function is linked with pre-agreed parameters which have to be triggered in order to start accumulating losses. Triggered points can be structured in many, and different, ways from a sliding scale of actual losses experienced by the issuer (indemnity) to a trigger which is activated when industry wide losses from an event hit a certain point (industry loss trigger) to an index of weather or disaster conditions of a certain severity that will trigger wide-spread cover-side losses (as we later describe; see Hagedorn et al. (2009), Burnecki et al. (2011) and Shao (2015)). The indemnity trigger type is subject to the highest degree of moral hazard due to the fact that loss is controlled by sponsor. To tackle this problem, a better choice would be using an industry loss trigger or a parametric index trigger, although these might bear a relatively higher basis risk.

\subsection{Theoretical Developments in the Design and Pricing of CAT Risk Bonds}

Despite the increasing popularity in this area, the number of the existing studies devoted to CAT risk bonds is relatively small. With much of this work developed to theory, at the expense of practical application, i.e. to provide a tradeable CAT risk bond for a given catastrophe. We aim to do both in the following sections. These current sections review the existing literature on CAT risk bonds development.

The implementation of catastrophe risks requires an incomplete markets framework to price the bonds, because the catastrophe risks cannot be replicated by a portfolio of primitive securities, see Harrison and Kreps (1979), Cox et al. (2000), Cox and Pedersen (2000), Vaugirard (2003),

\footnotetext{
${ }^{3}$ http://www.artemis.bm/deal_directory/cat_bonds_ils_issued_outstanding.html. ARTEMIS is an online website since 1999, Artemis provides news, analysis and data on catastrophe bonds, insurance-linked securities and alternative reinsurance capital
} 
etc.. It is known, from Young (2004) and elsewhere, that there is no universal pricing theory that successfully addresses issues such as specification of hedging strategies and price robustness under the incomplete markets framework. Thus, different interesting approaches have been derived in the literature. For instance, Wang (2004) addresses the market incompleteness using the Wang transform, an approach which has been adopted by Lin and Cox (2005, 2008), Pelsser (2008), Galeotti et al. (2011). Froot and Posner (2000, 2002) derive an equilibrium pricing model for the uncertain parameters of multi-events risks. A more theoretical approach is proposed by Föllmer and Schweizer (1991), who introduce a minimal martingale measure for the option pricing, whereas Schweizer (1995) uses a variance optimal martingale measure.

1.3.1. Indifference and Equilibrium Pricing Approaches. Another common technique used in an incomplete market setting is based on the principle of equivalent utility for obtaining indifferent pricing. This popular approach is inherited in the present paper. In actuarial science, and particularly, in the area of CAT risk bonds, Young (2004) calculates the price of a contingent claim under a stochastic interest rate for an exponential utility function. Her paper is further extended by Egami and Young (2008), who introduce a more complex payment structure based also on the utility indifference approach. Dieckmann (2011) applies a CAT risk bond model based on the consumption, while Zhu (2011) details the premium spread using an intertemporal equilibrium framework. Braun (2012) analyses the premium using OLS regression with robust standard errors. In the very interesting and applicable approach by Cox and Pedersen (2000), a time-repeatable representative agent utility is used. Their approach is based on a particular term structure model for the interest rates and a probabilistic framework for the catastrophe risks. They assume that the agent uses a utility function to make choices about consumption streams. Their theoretical results are applied to Morgan Stanley, Winterthur, USAA, and Winterthur-style bonds. Reshetar (2008) uses similar settings for multiple-event CAT risk bonds. Zimbidis et al. (2007) adopt the Cox and Pedersen (2000) framework to price a Greek risk bond for earthquakes using the equilibrium pricing theory with dynamic interest rates. Recently, Cox and Pedersen (2000)'s approach has been extended further by Shao et al. (2015a) in a multi risk environment improving simultaneously the results proposed by Zimbidis et al. (2007).

1.3.2. Continuous-time No arbitrage Models. Baryshnikov et al. (2001) present a continuous time no-arbitrage price of zero coupon and non-zero coupon CAT risk bonds that incorporates a compound doubly stochastic Poisson process. The discussions of this paper has been further developed in Burnecki and Kukla (2003) where the authors assume that the arbitrage and real world measures coincide. Using the PCS database, their results are applied to calculate the arbitrage-free price of zero-coupon and coupon CAT bonds. Burnecki et al. (2011) illustrate the value of CAT risk bonds with loss data provided also by PCS, when the flow of events is an inhomogeneous Poisson process. These approaches are utilized by Härdle and Cabrera (2010) for calibrating CAT risk bonds prices for Mexican earthquakes. Jarrow (2010) obtains a simple closed form CAT risk bond solution with a LIBOR term structure of interest rate.

Another approach in continuous time is to model the trigger involving aggregate loss process. It is important to note that Vaugirard (2003) is the first to develop a simple arbitrage approach for evaluating catastrophe risk insurance-linked securities, although they employ a non-traded underlying framework. In that paper, CAT bondholders have a short position on an option. Lin et al. (2008) apply a Markov-modulated Poisson process for catastrophe occurrences using a similar approach to that of Vaugirard (2003). Lee and Yu $(2002,2007)$ introduce the default risk, moral hazard and basis risk with stochastic interest rate. Pérez-Fructuoso (2008) develops a CAT bond with index triggers. Ma and Ma (2013) propose a mixed approximation method to simplify the distribution of aggregate loss and to find the numerical solutions of CAT bonds with general pricing formula. In addition, Nowak and Romaniuk (2013) expand Vaugirard's model and obtained CAT 
bond prices using Monte Carlo simulations with different payoff functions and spot interest rates. Recently, in Shao et al. (2015b), a two-dimensional semi-Markov process are used and an analytical bond pricing formula is derived in a stochastic interest rate environment with aggregate claims that follow compound forms, where the claim inter-arrival times are dependent on the claim sizes. In this approach different payoff functions have been implemented. It should be mentioned that in Bilal et al. (2015), a two-coverage type trigger nuclear catastrophe (N-CAT) risk bond for potentially supplementing the covering of US commercial nuclear power plants beyond the coverage per the Price Anderson Act as amended, and potentially other plants worldwide is proposed and designed. The N-CAT peril is categorized by three risk layers: incident, accident and major accident. The pricing formula are derived by using a semi-Markovian dependency structure in continuous time.

1.3.3. Agriculture-related CAT Risk Bonds. Research and developments on agriculture related CAT risk bonds has been more limited. Sun et al. (2015) design a CAT bond, based upon the catastrophic failure of long and short rains in Kenya with indemnity tranches for failure in either or both rains, with the recommendation that CAT bonds of this type be issues as a component of sovereign debt. Vedenov et al. (2006) design a zero-coupon CAT bond for Georgian (USA) cotton triggered by state-wide multiple-peril yield loss. These structures can substitute for reinsurance where agricultural reinsurance markets are not developed, or can complement (offset) reinsurance claims where they do (Duncan and Myers (2000)). Turvey (2008) explores pricing weather-linked bonds but more in the context of businesses with weather sensitive risks. Likewise there have been a number of papers written on commodity-linked bonds which are bonds issued with option or option-like structures linked to the price of a specific commodity; (see, for instance, Harrison and Kreps (1979), Cox and Schwartz (1982), O'Hara (1984), Carr (1987), Gibson and Schwartz (1990), Schwartz (1997), Miltersen and Schwartz (1998), Turvey (2006), etc). While a precursor to the sort of bond structures discussed in the current paper all of the above focused on corporate structures and tradable financial products such as commodity options.

In this present paper, our approach can be distinguished in several different ways from the existing literature. First of all the utility indifference pricing method we use for the CAT bonds is from the insurance company point of view. For that reason our framework is not dynamic since the insurance company will write the contract upfront i.e., the terms of the CAT bond is agreed today and the liabilities will be delivered at the end of the contract (e.g., 3 or 5 years). The other characteristic of our proposed framework is that our contract is OTC, which means is not traded on a usual basis in the market. The price we find in this setting can be interpreted as the insurance company's evaluation from the CAT risk bond in its balance sheet. Finally, our approach is interesting from economical point of view, since utility indifference pricing is a popular method for pricing insurance contracts in economics textbooks, while we are using it in this paper for pricing CAT bonds.

The rest of the paper is organized as follows. In Section 2, the model formulation is proposed. In our modeling framework, the insurance company and a hedge-fund are incorporated. Then, the final wealth of the insurance company is investigated if CAT and no CAT risk bonds is issued. Then, the price for risk neutral and risk averse insurance companies are presented in Section 3. The numerical simulations and the price of an AgriCAT bond using real data from two different cities in Iran are discussed in Section 4. Section 5 concludes the paper.

\section{Model Formulation}

In this section the model formulation is proposed and discussed. First, a discrete-time, probabilistic framework is considered. The model consists of two distinct periods of time 0 and $T$, representing the beginning and the end of the insurance contract. $\Omega=\left\{\omega_{1}, \ldots, \omega_{n}\right\}$ represents the set of all scenarios associated with different tranches of a catastrophic event, for different drought intensities 
realized at time $T$. Scenario $\omega_{i}$ occurs with probability $p_{i}>0$, where $\sum_{i=1}^{n} p_{i}=1$ and $\mathrm{E}(\cdot)$ represents the expected value. The notation that is used in the sequel is defined in Table 2:

Table 1. The necessary notations for CAT risk bonds

\begin{tabular}{llll}
\hline$w:$ & the insurance company's initial wealth & $Y:$ & the catastrophe loss variable \\
$X:$ & the hedge fund pay-off at time $T$ & $r:$ & the market bind interest rate \\
$L I:$ & the LIBOR interest rate & $E:$ & excess to LIBOR \\
$B:$ & the bond value at time 0 & $C:$ & the CAT bond value at time 0 \\
$\pi:$ & $\mathrm{R}^{n} \rightarrow \mathrm{R}_{+}:$the risk premium function & $u:$ & $\mathrm{R}_{+} \rightarrow \mathrm{R}:$ the insurance company utility function \\
\hline
\end{tabular}

At this point two random variables $X, Y$ need to be introduced. $X$ is linked to the hedge-fund company and $Y$ to the CAT risk bond itself. In particular, we have the following:

$$
Y=\left\{\begin{array}{lc}
y_{1}, & \text { with probability } p_{1} \\
\vdots & \vdots \\
y_{n}, & \text { with probability } p_{n}
\end{array}\right.
$$

where $\left\{y_{i}\right\}_{i=1}^{n}$ is the set of the various payoffs to the client, with respect to the intensity of the event which occurs with probability $\left\{p_{i}\right\}_{i=1}^{n}$. We can view $\left\{y_{i}\right\}_{i=1}^{n}$ as the different tranches of the CAT risk bond. The higher the intensity of a catastrophic event is, the smaller the probability of occurrence and the higher the payoff will be. The spectrum of the CAT risk bond under which it will be triggered is explicitly stated at time 0 . The intensity can be measured by an index; for instance, Richter scale for earthquake or the water dropped level in $\mathrm{cm}$. in a specific period or Celsius degrees for temperature related to the structure of the CAT risk bonds (see Cox and Pedersen (2000) and Shao et al. (2015a)). Usually the duration of the CAT risk bond is from 1 to 5 years. The excess $E$ over the LIBOR rate $L I$ is given as a risk premium, in order to make the CAT risk bond an attractive investment.

Let $1=S_{1}>S_{2}>\cdots>S_{n}=0$ denote the set of bond recovery for the hedge-fund. Note that the smaller the intensity is, the higher the proportion will be. We introduce the recovery variable as follows

$$
S=\left\{\begin{array}{lc}
S_{1}, & \text { with probability } p_{1} \\
\vdots & \vdots \\
S_{n}, & \text { with probability } p_{n} .
\end{array}\right.
$$

Therefore, we introduce the hedge-fund payoff at time $T$,

$$
X=(L I+E+S) C .
$$

We also need to clarify that in the case of large scale catastrophic events the interest or even the principal is usually forgone.

In our modeling framework, we have two major players: an insurance company and a hedgefund. We assume that the insurance company behaves according to a utility function, and makes decisions in order to maximize its expected utility. Therefore, we consider that the insurance company determines the price of the CAT risk bond within the utility indifference pricing method. In general, this method is based on the following procedure; see, for instance, Monoyios (2008) and Carmona (2009). Given two strategies 1 and 2, we need to specify an amount of money $h$ to be given to the insurance company in order to make it indifferent between choosing strategies 1 or 2 . In other words, the optimal utility in the two strategies has to be equal. In particular, when pricing a CAT risk bond, the insurance company has two strategies: either to issue a CAT risk bond (in 
order to diversify away part of the risk towards the financial market by transferring the risk to the hedge fund) or not. In the following, we find the optimal value of the utility at each case and equalize them together.

On the other hand, the hedge-fund is assumed to behave like a firm and it won't enter the business if the expected net profit is non-negative. It is clear that the net profit condition is as follows:

$$
N=L I+E+S-(1+r) .
$$

The expected net profit is $\mathrm{E}(N)=L I+E+\mathrm{E}(S)-(1+r)$.

Our assumption is that the hedge fund will enter the business only if

$$
\mathrm{E}(N) \geq 0
$$

In other words, for a hedge-fund to participate to the transactions, the expected recovery of one unit of the CAT risk bond is not less than the zero-coupon interest rate. We also call the condition $\mathrm{E}(N)=0$, the no-arbitrage condition. Note that even if $\mathrm{E}(N)>0$ it should be a very small number, otherwise the business is a very good deal, and indeed an arbitrage.

\subsection{Utility without CAT risk bond}

In this part of the paper, the final wealth of the insurance company is investigated if no CAT risk bond is issued. In the following figure, the transactions is illustrated when the insurance company does not issue any CAT risk bond.

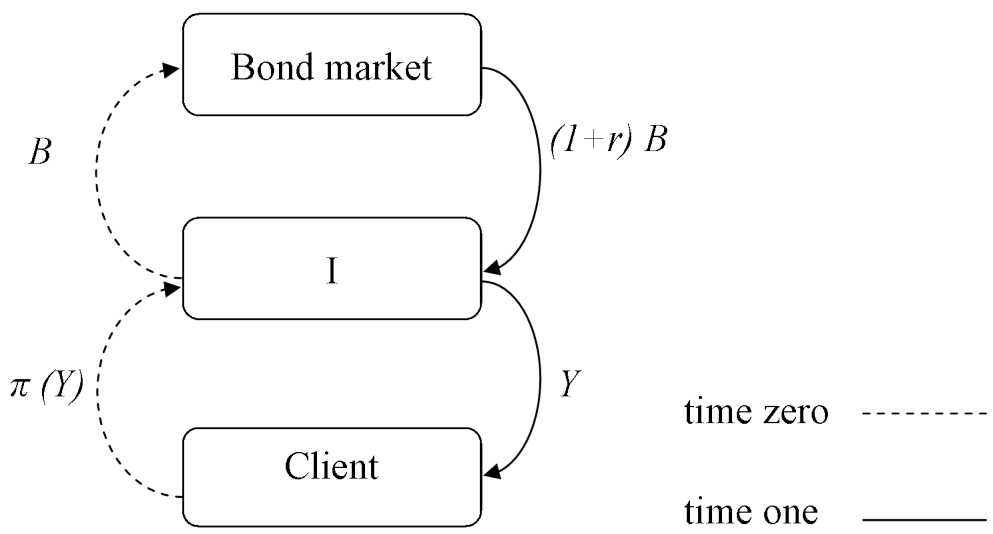

Figure 2. No CAT risk bond transaction. The insurance company receives premium and pays back $Y$ to the client.

The dashed lines are transactions at time 0 and the solid ones at time $T$. As one can see at time $0, \pi(Y)$ is paid to the insurance company for undertaking the risk. With the aggregate endowment $W=\pi(Y)+w I$ buys $B$ value of the zero-coupon bonds, which at time $T$ will pay off at the rate $r$. At time $T, I$ will receive the face value $(1+r) B$ and has to cover the loss $Y$. Given that the terminal wealth is $W_{T}=(1+r) B-Y$ the insurance company's utility for the initial endowment 
$w$ is equal to ${ }^{7}$

$$
U(W)=\mathrm{E}(u[(1+r) W-Y])=\sum_{i=1}^{n} p_{i} u\left((1+r) W-y_{i}\right)
$$

By introducing the final payoff as $L(W)=(1+r) W-Y$, we may rewrite $U(W)$ as follows,

$$
U(W)=\operatorname{E} u(L(W))
$$

\subsection{Utility with CAT risk bonds}

In this part of the section, the expected terminal wealth of the insurance company is maximized in the case that a CAT risk bond is issued. The main difference with the previous part is that the insurance company is allowed to diversify the risk away by transferring it into a hedge fund. The following figure summarizes the transactions, see Figure 3.

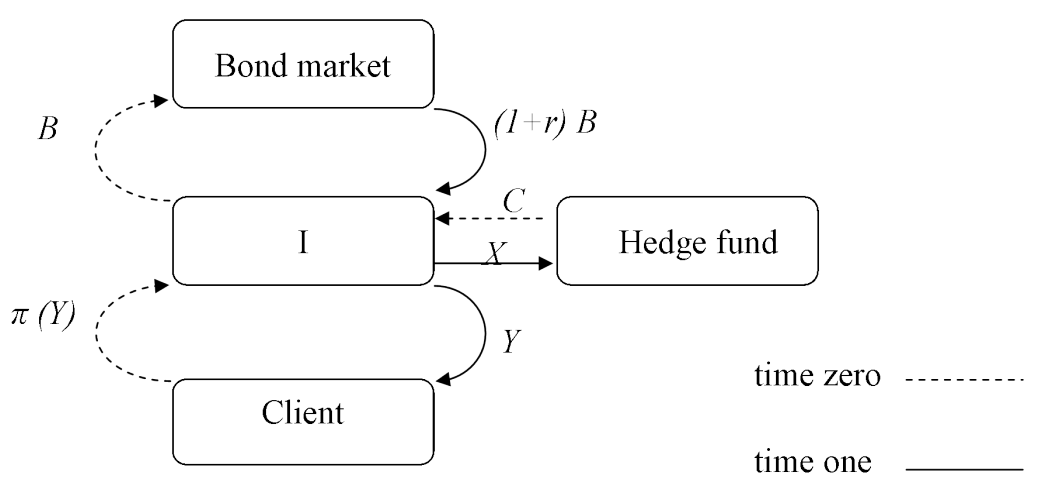

Figure 3. CAT bond transaction. The reinsurance company receives premium and pays back $Y$. At the same time another transaction with a hedge fund takes place.

Like in the previous part, at time 0 , the insurance company receives premium $\pi(Y)$ from the client. Additionally, the insurance company receives an amount $C$ by issuing and selling a CAT risk bond to the hedge-fund. The total amount will be used to buy $B$ value of a zero-coupon at rate $r$. At time $T$, the insurance company will receive $(1+r) B$ from the zero-coupon bond, and has to recover the loss $Y$. Furthermore, the insurance company has to pay $X$, i.e. LIBOR + Excess percent of $C$ to the hedge-fund plus a portion of $C$ to the hedge-fund. Therefore, the insurance company maximizes its utility finding the optimal value of $C$,

$$
V(W)=\max _{(B, C) \in A_{w}} \mathrm{E}\left(u\left(W_{T}\right)\right)
$$

over the set of all admissible controls $A_{W}=\{(B, C): B=C+W\}$. Let $W_{T}=(1+r) B-(Y+X)$

${ }^{7}$ Probably one would expect to solve the following maximization problem

$$
\max _{\{B: B=\pi(Y)+w\}} \mathrm{E}(w((1+r) B-Y))
$$

to find the value. However when replacing $B$ we do not have any maximization. 
be the insurance company's terminal wealth. Given that $B=C+W$, we have

$$
\begin{aligned}
V(W) & =\max _{C} \mathrm{E} u[(1+r)(C+W)-(Y+X)] \\
& =\max _{C} \mathrm{E} u[L(W)-N C] \\
& =\max _{C} \sum_{i=1}^{n} p_{i} u\left[L_{i}(W)-N_{i} C\right] .
\end{aligned}
$$

In order to solve this problem, let us introduce the function

$$
g(C)=\mathrm{E} u[L(W)-N C] .
$$

If we denote the optimal value of the CAT risk bond by $C(W)$ then $C(W)$ has to solve the following problem

$$
\frac{d g}{d C}(C(W))=0
$$

or equivalently,

$$
\mathrm{E}\left[N u^{\prime}(L(W)-N C(W))\right]=0 .
$$

Following all the notations above, for an initial wealth $w$, the indifference bid and ask prices $h_{b i d}=h_{b i d}(W)$ and $h_{a s k}=h_{a s k}(W)$ respectively, are the solutions of the following problem

$$
V(W)=U\left(W+h_{a s k}\right)
$$

and

$$
V\left(W-h_{b i d}\right)=U(W) .
$$

In particular, we have to solve the following equations in order to find bid and ask prices

$$
\mathrm{E}[u(L(W)-N C(W))]=\mathrm{E}\left(L\left(W+h_{a s k}\right)\right)
$$

and

$$
\mathrm{E}\left[u\left(L\left(W-h_{b i d}\right)-N C\left(W-h_{b i d}\right)\right)\right]=\mathrm{E}(L(W)) .
$$

The solution of the above equations with respect to $h$ provides a pricing formula for the CAT risk bond in the following sense. The ask price $h$ is the amount of money paid to the insurance company under which it will be indifferent on whether the CAT risk bond will be issued or not. Respectively, the bid price is the amount which it is willing to pay in order to avoid the risky situation.

At this point, we justify the bid and ask price that we introduced in this paper in more details. In order to find bid and ask prices, we have to specify two things. First, we have to know what is the commodity that is traded in the market, second, we have to know the role of the agent, i.e., if it is buyer or seller. As it is clear from the setup, in our problem, risk is the thing that is transferred. In other words the insurance company has a demand for an 'anti-risk' product; which roughly speaking one would say the anti-risk is traded. On the other hand, the insurance company 
is willing to buy this product, which puts it at the role of a buyer. Now, we know that bid price is the maximum amount that one would pay to buy a commodity. Translating it into our framework, this is the maximum amount that the insurance company is willing to pay to have the CAT risk bond. This is where the insurance company moves from a riskier situation to less risky one.

So let us assume that in the first situation the aggregate endowment is equal to $W$. Then to find the bid price, one needs to ask how much the insurance company is willing to pay in order to have the same utility i.e., what is $h$ that $U(W)=V\left(W-h_{b i d}\right)$. In the same way one can justify the ask price in the definition above. Note that is $U$ and $V$ are invertible then the ask and bid prices are accessible through the following relations

$$
h_{a s k}=U^{-1}(V(W))-W \text { and, } h_{b i d}=W-V^{-1}(U(W)) .
$$

Finally, in order to find the demand curve of the CAT risk bond market we have to consider percentages of the CAT bond. If we consider the insurance company will trade a $k$ - percentage of the CAT bond, where $k \in[0,1]$, then its terminal wealth is $W_{T}=(1+r) B-k(Y+X)$ and optimization is defined by

$$
V(w ; k)=\max _{(B, C) \in A_{w}} \mathrm{E}\left(u\left(W_{T}\right)\right) .
$$

Let the final payoff to the client be $L(w)=(1+r)(k \pi(Y)+w)-k Y$ then since $B=k C+k \pi(Y)+w$ we obtain that

$$
V(w ; k)=\max _{C} \mathrm{E} u[L(w ; k)-k N C]
$$

The ask and bid prices are the solutions of the following equations, respectively.

$$
\mathrm{E}[u(L(w ; k)-k N C(w))]=\mathrm{E}(L(w+h ; k))
$$

and

$$
\mathrm{E}[u(L(w-h ; k)-k N C(w-h))]=\mathrm{E}(L(w ; k)) .
$$

The above formulations has the advantage that it can be used to find the demand curve for a CAT risk bond.

\section{Particular Cases}

We can consider two general cases, first when the insurance company is risk neutral and second when the insurance company is risk averse.

\subsection{Risk neutral and risk avers insurance company}

Now we have to look at two cases: first when the CAT bond is not issued by the insurance company, the utility will be

$$
\mathrm{E}(L(W))
$$


and secondly, when the CAT bond is issued

$$
\max _{C} \mathrm{E}[L(W)-N C]=\mathrm{E}(L(W))+\max _{C}(-\mathrm{E}(N) C) .
$$

One can easily see that the solution exists if and only if $\mathrm{E}(N) \geq 0$. In the case that the positive net profit principle holds then $C(w)=0$ and we have no CAT bond to price whereas if the no-arbitrage holds, i.e., $\mathrm{E}(N)=0$, then price can be set at any number.

Now, we consider a risk averse insurance company. We begin our analysis by considering that the insurance company decides according to a quadratic utility, as the most simple utility in the literature, see Gerber and Pafum (1998), Pantelous and Passalidou (2015) (and references therein). Quadratic utility functions are usually used as an approximation to more complex utility functions and are very helpful because of the insight they provide and due to optimality of the mean-variance analysis. It should be mentioned here that in practice, the quadratic utility is not so popular because it is characterized by an increasing absolute risk aversion. In our case, without any loss of generality, it can benefit us with some interesting findings. This utility function can be expressed as follows

$$
u(x)=x-b x^{2},
$$

for a small $b$, which measures the risk tolerance of the insurance company. Since any utility function needs to have a non-negative derivative we need to accept the restraint ${ }^{8}$ :

$$
x \leq \frac{1}{2 b} .
$$

Next, we calculate $C(W)$. Easily since $u^{\prime}(x)=1-2 b x$ and replacing in (2) we get

$$
\mathrm{E}[N(1-2 b(L(W)-N C(W)))]=0 .
$$

By direct calculations, we get

$$
C(W)=\frac{2 b \mathrm{E}(N L(W))-\mathrm{E}(N)}{\mathrm{E}\left(N^{2}\right)} .
$$

Given that $L(W)=(1+r) W-Y$, we will have

$$
C(W)=\beta+\alpha W,
$$

where

$$
\alpha=\frac{2 b(1+r) \mathrm{E}(N)}{\mathrm{E}\left(N^{2}\right)} .
$$

and

$$
\beta=\frac{-2 b \mathrm{E}(N Y)-\mathrm{E}(N)}{\mathrm{E}\left(N^{2}\right)} .
$$

As one can see according to the net profit condition (1), $\alpha$ is a non-negative number, and therefore $C(W)$ is a linear and non-decreasing function of $W$. Using the notation above, one can rewrite

${ }^{8}$ This is due to fact that utility increases at a diminishing rate. 
$V(W)$ as

$$
V(W)=\max _{C} \mathrm{E}\left[L(W)-b L(W)^{2}\right]+C \mathrm{E}[2 b L(W) N-N]-b C^{2} \mathrm{E}\left[N^{2}\right]
$$

Since this expression is quadratic in terms of $C$, the maximum value is equal to ${ }^{1}$

$$
V(W)=\mathrm{E}\left[L(W)-b L(W)^{2}\right]+\frac{(\mathrm{E}[2 b L(W) N-N])^{2}}{4 b \mathrm{E}\left[N^{2}\right]}=U(W)+\frac{(\mathrm{E}[2 b L(W) N-N])^{2}}{4 b \mathrm{E}\left[N^{2}\right]}
$$

Let us now assume that $E(N)=0$. In that case, we have

$$
\begin{aligned}
& \alpha=\frac{2 b(1+r) \mathrm{E}(N)}{\mathrm{E}\left(N^{2}\right)}=0, \\
& \beta=\frac{-2 b \mathrm{E}(N Y)}{\mathrm{E}\left(N^{2}\right)}, \\
& V(W)=U(W)+\frac{(\mathrm{E}[2 b((1+r) W-Y) N-N])^{2}}{4 b \mathrm{E}\left[N^{2}\right]}=U(W)+\frac{b(\mathrm{E}[Y N])^{2}}{\mathrm{E}\left[N^{2}\right]} .
\end{aligned}
$$

First of all (5) and (6) show that if there is no arbitrage for the hedge fund then the insurance company's initial wealth and the insurance premium do not have any effect on the number of traded CAT bonds. Second, (6) indicates that from the insurance company's point of view in order to have a business, $\mathrm{E}(N Y) \leq 0$ has to hold. Indeed, this way the net profit is canceling out the adverse impact of the loss variable. Third, from (7) one can see that the first derivatives of $V$ and $U$ are equal i.e., $V^{\prime}=U^{\prime}$, which shows there is no difference in the risk taking appetite with or without CAT bonds. Furthermore, this will help us in the following to find the evolution of the ask and bid prices with respect to the changes in the insurance company's wealth.

Since $U$ and $V$ are utilities, they are non-decreasing with non-increasing derivatives. Using these facts along with $V^{\prime}=U^{\prime}$ and (3) we have

\footnotetext{
${ }^{1}$ Indeed the maximum value of $f(x)=a_{1} x^{2}+a_{2} x+a_{3}$ for a negative $a_{1}$ is equal to $a_{3}-\frac{a_{2}^{2}}{4 a_{1}}$.
} 


$$
\begin{aligned}
V(W) & =U(W)+\frac{b(\mathrm{E}[Y N])^{2}}{\mathrm{E}\left[N^{2}\right]} \Rightarrow V(W) \geq U(W) \\
& \Rightarrow W \geq V^{-1}(U(W)) \\
& \Rightarrow U^{\prime}(W) \leq V^{\prime}\left(V^{-1}(U(W))\right) \\
& \Rightarrow \frac{U^{\prime}(W)}{V^{\prime}\left(V^{-1}(U(W))\right)} \leq 1 \\
& \Rightarrow \frac{d\left(V^{-1}(U(W))\right)}{d W} \leq 1 \\
& \Rightarrow \frac{d\left(V^{-1}(U(W))-W\right)}{d W} \leq 0 \\
& \Rightarrow \frac{d\left(-h_{b i d}\right)}{d W} \leq 0 \\
& \Rightarrow \frac{d\left(h_{b i d}\right)}{d W} \geq 0 .
\end{aligned}
$$

With very similar argument one can also see that $\frac{d\left(h_{a s k}\right)}{d W} \geq 0$. This means that if the insurance company uses a quadratic utility function in a market that there is no arbitrage for the hedge fund then, the ask and bid prices are increasing in terms of the the insurance company's initial wealth and insurance premium.

\section{AgriCAT Risk Bond}

The financial economists' interest in CAT risk bonds applied to problems of agriculture stems from the observable fact that when natural catastrophes occur the covariate risk is spread among many, often very poor, farmers that cannot rely on traditional self-insuring diversification-savings strategies to mitigate risk. Nor can crop or other forms of agricultural insurance develop when infrequently, but with probability greater than zero, an event will arise with losses so great that the capital of the insurer is inadequate to cover. A study by the World Bank concluded that the role of the international development community is critical to providing access to catastrophe risk markets and expanding the range of risks covered, Cummins and Mahul (2009). Even when government sponsored (as in USA and Canada) or global reinsurance markets are present the transfer of large systemic risks in the form of marketable catastrophe bonds can serve as an efficient and effective risk transfer mechanism. Lesser developed or developing countries that are highly reliant on agriculture often have poorly developed financial and risk-transfer/insurance markets or face catastrophic risks that cannot easily be absorbed by an un-diversified economy, Skees and Barnett (1999, 2006), Miranda and Vedenov (2001) and so it may be to the State's advantage to issue CAT bonds to protect treasuries, maintain current accounts and provide emergency relief. These actions depend on assessing the government's contingent liability to natural disasters; enabling risk transfer to competitive insurance and reinsurance market; and financing sovereign risk, see Cummins and Mahul (2009).

In this section, we apply the proposed method of Section 2, to calculate the ask and bid prices of CAT risk bonds related to agricultural catastrophes. We use data for two different cities in Iran, Mashhad and Tabriz, in order to find the AgriCAT risk bond prices of a natural catastrophe which hits the harvest in these two cities. We also look at a risk management problem by diversifying the risk of a portfolio of AgriCAT risk bonds across these two cities. 
In particular, using monthly climate data, we calculate probabilities of catastrophic events related to low temperatures and their consequences in the agriculture sector. Mashhad is in the north-east of Iran at 1,000 meters altitude with daily temperature from 1.5 degrees Celsius in January to 26 degrees Celsius in July. In general Mashhad has a cold semi-arid steppe climate. Tabriz is located in the north-west at 1,350 meters above sea level with average daily temperature at -4 degree Celsius in January to 26 degree Celsius in July. It has a semi-arid climate with dry summers.

Cold damage varies a lot between different kind of crops. Damage that occurs above 0 degree Celsius is chilling injury while below 0 degree Celsius is freezing one. Most crops can survive from a low freezing damage but not from a high.

For reasons of clarity and without loss of generality, we constrain ourselves to the case of a binomial type catastrophic event with no CAT risk bond tranches issued. Therefore, if a catastrophe occurs the bond is triggered, the client is paid and in the hedge fund principal is totally forgiven i.e. the loss for the hedge fund is complete.

We use historical monthly data from 1951-2014, from the National Centers for Environmental Information, National Oceanic and Atmospheric Administration ${ }^{9}$ (NOAA) to calculate the probabilities of the temperature dropping below a certain threshold, which is used as a triggering point of the AgriCAT risk bond.

The most critical period is prior to harvest and therefore, we only use data obtained for March and in particular we use average low temperatures, since the greatest temperature damage occurs during the night where the lowest temperatures are observed. It has been suggested that weather temperature within a year follows Beta distribution, (Snyder and Melo-Abreu (2005)), but we observed that the temperature for March follows normal distribution. This opinion is enhanced by the following QQ-plots and verified by the chi-squared test. The chi squared value is 3.0025 and 7.2760 for Mashhad and Tabriz, respectively, with rejection of normality being 11.0705.
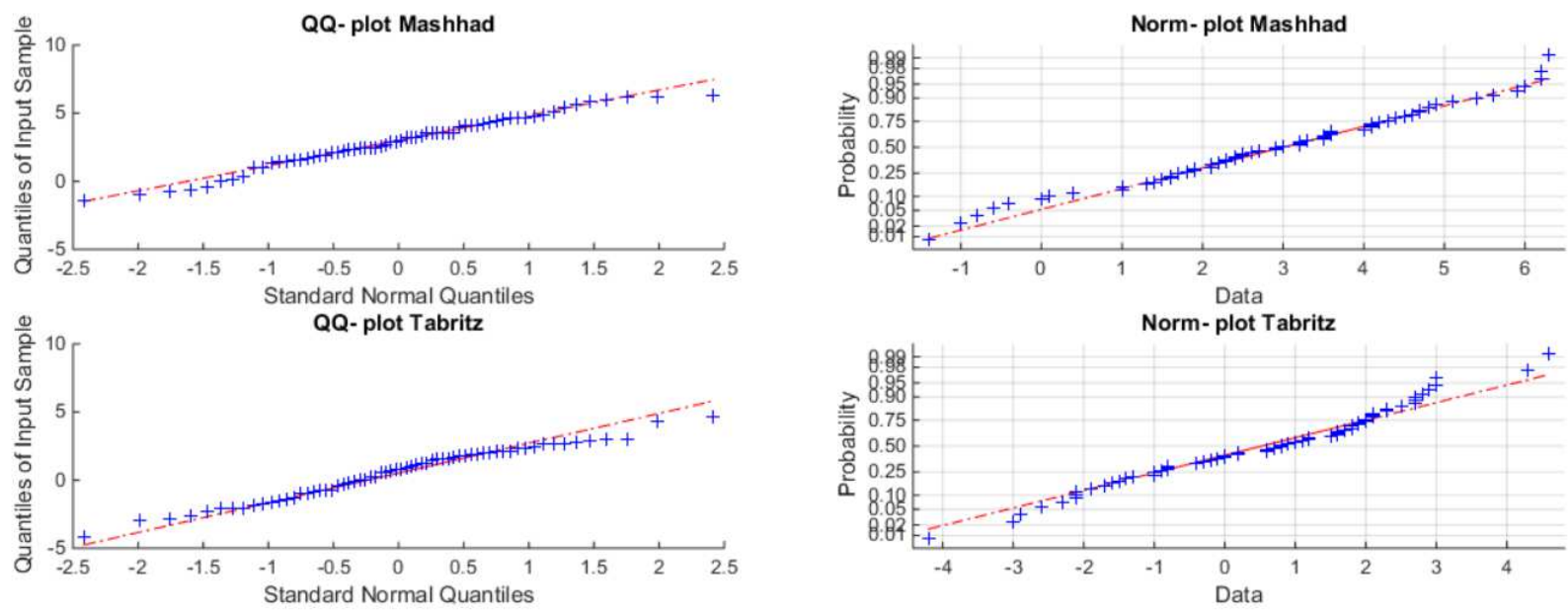

Figure 4. QQ and Normal Plots for Mashhad and Tabriz.

Fitting the monthly data, we obtain the maximum likelihood estimators for the mean and standard deviation for both cities, as shown in Table 2.

Setting the temperature threshold at -7 degree Celsius, which is quite low, but reasonable since we investigate total catastrophic events, using Table 2 we calculate the probabilities of the temperature dropping below the threshold in those cities which are given in Table 3.

We use Table 3, to calculate ask and bid prices for various levels of initial wealth, as well as different percentages of AgriCAT risk bonds in order to graph the demand curve. For the remaining

${ }^{9}$ See https://www.ncei.noaa.gov/ 
Table 2. Mean and Standard Deviation

\begin{tabular}{lll} 
& Mashhad & Tabriz \\
$\hat{\mu}$ & 2.9094 & 0.5281 \\
$\hat{\sigma}$ & 1.8657 & 1.9010 \\
\hline
\end{tabular}

Table 3. Catastrophic Probabilities

\begin{tabular}{lll}
\hline & Mashhad & Tabriz \\
Probabilities & 0.00221 & 0.01862 \\
\hline
\end{tabular}

calculations, we use an isoelastic utility function

$$
u(x)=\frac{x^{1-\eta}-1}{1-\eta} .
$$

In general power utility function has a number of desirable properties, (see Arrow (1971) and Wakker (2008)), including constant relative risk aversion (CRRA). This means that the relative risk aversion parameter defined by $-\frac{x u^{\prime \prime}(x)}{u^{\prime}(x)}$ is equal to constant $\eta$. The parameter $\eta$ shows the level of relative risk aversion of the insurance company i.e., larger $\eta$ means that the insurance company is risk averse, while $\eta=0$ is associated with the case that the insurance company is risk neutral.

In the tables 4 and 5, we display the ask and bid prices for the AgriCAT risk bonds for different percentages $k=20,40,60,80$ and $100 \%$ of a unit of a AgriCAT risk bond. For the calculations we use the general formulas of the Section 2.2 combined with Eq. (8). In the Figure 4, we view the demand curve for the CAT risk bond for the various percentages.

Table 4. Ask and Bid Prices for Mashhad

\begin{tabular}{lllll}
\hline Wealth & 2000 & 3000 & 4000 & 5000 \\
& \multicolumn{4}{c}{ Ask Prices } \\
$\mathrm{k}=0.2$ & 73.5877 & 112.9499 & 152.3121 & 191.6743 \\
$\mathrm{k}=0.4$ & 68.4512 & 107.8134 & 147.1755 & 186.5377 \\
$\mathrm{k}=0.6$ & 63.3148 & 102.6768 & 142.0390 & 181.4011 \\
$\mathrm{k}=0.8$ & 58.1784 & 97.5403 & 136.9024 & 176.2645 \\
$\mathrm{k}=1$ & 53.0420 & 92.4039 & 131.7659 & 171.1280 \\
\multicolumn{5}{c}{ Bid Prices } \\
$\mathrm{k}=0.2$ & 70.8009 & 108.6723 & 146.5438 & 184.4153 \\
$\mathrm{k}=0.4$ & 65.8588 & 103.7303 & 141.6018 & 179.4733 \\
$\mathrm{k}=0.6$ & 60.9170 & 98.7883 & 136.6598 & 174.5312 \\
$\mathrm{k}=0.8$ & 55.9751 & 93.8463 & 131.7177 & 169.5891 \\
$\mathrm{k}=1$ & 51.0333 & 88.9045 & 126.7758 & 164.6472 \\
\hline
\end{tabular}

Remark 1 As one can see, the CAT bond prices increase when the initial wealth is larger. This is in opposite direction to what we have analytically studied with the quadratic utility function. As it was mentioned earlier, this is because the quadratic utility is increasing relative risk averse, while CRRA utility is constant relative risk averse.

In the sequel, we investigate the construction of a portfolio of AgriCAT risk bonds for the above two cities. For this, we calculate the joint probabilities for catastrophic or not events. We use 
Table 5. Ask and Bid Prices for Tabriz

\begin{tabular}{lllll}
\hline Wealth & 2000 & \multicolumn{4}{c}{3000} & 4000 & 5000 \\
Ask Prices \\
$\mathrm{k}=0.2$ & 41.5555 & 63.7869 & 86.0183 & 108.2497 \\
$\mathrm{k}=0.4$ & 38.6488 & 60.8798 & 83.1110 & 105.3423 \\
$\mathrm{k}=0.6$ & 35.7427 & 57.9732 & 80.2041 & 102.4353 \\
$\mathrm{k}=0.8$ & 32.8373 & 55.0670 & 77.2976 & 99.5285 \\
$\mathrm{k}=1$ & 29.9327 & 52.1613 & 74.3913 & 96.6220 \\
\multicolumn{5}{c}{ Bid Prices } \\
$\mathrm{k}=0.2$ & 40.6518 & 62.3996 & 84.1476 & 105.8955 \\
$\mathrm{k}=0.4$ & 37.8083 & 59.5558 & 81.3035 & 103.0514 \\
$\mathrm{k}=0.6$ & 34.9655 & 56.7124 & 78.4599 & 100.2076 \\
$\mathrm{k}=0.8$ & 32.1233 & 53.8695 & 75.6166 & 97.3640 \\
$\mathrm{k}=1$ & 29.2819 & 51.0270 & 72.7736 & 94.5207 \\
\hline
\end{tabular}

different portfolio weights $a$ and we calculate the expected return to the client, depending on the catastrophic event and the random variable $Y$. We denote the premium depending on $a$ by $\pi(Y)_{a}$.

Table 6. Joint Probabilities

\begin{tabular}{ll}
\hline Catastrophic Event & Joint Probabilities \\
Mashhad \& Tabriz & $p_{1,1}=0.0000000361848688430048$ \\
Mashhad (only) & $p_{1,2}=0.0000000321474848930197$ \\
Tabriz (only) & $p_{2,1}=0.0000426181850481083$ \\
Neither Mashhad nor Tabriz & $p_{2,2}=0.999957313482598$ \\
\hline
\end{tabular}

Therefore, in order for the insurance company to have a profit, it is required that $\pi(Y)_{a}$ is greater than the expected pay-off. Similarly, we calculate the recovery variable $S$, for the same portfolio weights. For simplicity, we assume a binomial distribution for the recovery, i.e. either a total recovery or a complete loss.

Finally, using the probabilities of Table 6, we calculate the ask and bid prices of different percentages for different portfolio weights for a given initial endowment $W=2000$.

It is interesting to observe that the prices of the AgriCAT risk bonds are increasing with respect to the shares of Mashhad, meaning that the prices will increase if we increase the share of AgriCAT risk bond of Tabriz. This is quite understandable since Mashhad is a colder city and once Tabiz hits -7 degrees Celsius it is very likely that Mashhad also hits this temperature. In mathematical terms, if we denote the temperature of Tabriz by $T_{T}$ and Mashhad by $T_{M}$, then the probability of $P\left(T_{M}<-7 \mid T_{T}<-7\right)$ is almost one. This can shows that once the CAT bond is triggered in Tabriz, more likely it is also triggered in Mashhad, which is why a portfolio with $100 \%$ of Tabriz AgriCAT risk bond be less riskier and consequently cheaper. 
Table 7. Ask and Bid Prices for different portfolio weights

\begin{tabular}{|c|c|c|c|c|c|}
\hline Portfolio Weights & $0.1-0.9$ & $\begin{array}{r}0.3-0.7 \\
\text { Ask }\end{array}$ & $\begin{array}{l}0.5-0.5 \\
\text { Prices }\end{array}$ & $0.7-0.3$ & $0.9-0.1$ \\
\hline Portfolio Unit $\mathrm{k}=0.2$ & 77.8220 & 77.8386 & 77.8552 & 77.8718 & 77.8884 \\
\hline Portfolio Unit k=0.4 & 72.3906 & 72.4061 & 72.4216 & 72.4370 & 72.4525 \\
\hline Portfolio Unit k=0.6 & 66.9594 & 66.9737 & 66.9880 & 67.0023 & 67.0166 \\
\hline Portfolio Unit $\mathrm{k}=0.8$ & 61.5280 & 61.5413 & 61.5544 & 61.5675 & 61.5806 \\
\hline Portfolio Unit k=1 & 56.0968 & $\begin{array}{c}56.1088 \\
\text { Bid }\end{array}$ & $\begin{array}{l}56.1208 \\
\text { rices }\end{array}$ & 56.1327 & 56.1447 \\
\hline Portfolio Unit $\mathrm{k}=0.2$ & 74.7120 & 74.7273 & 74.7426 & 74.7579 & 74.7732 \\
\hline Portfolio Unit $\mathrm{k}=0.4$ & 69.4977 & 69.5120 & 69.5263 & 69.5405 & 69.5547 \\
\hline Portfolio Unit $\mathrm{k}=0.6$ & 64.2834 & 64.2966 & 64.3098 & 64.3230 & 64.3362 \\
\hline Portfolio Unit $\mathrm{k}=0.8$ & 59.0692 & 59.0813 & 59.0934 & 59.1055 & 59.1177 \\
\hline Portfolio Unit k=1 & 53.8550 & 53.8660 & 53.8771 & 53.8881 & 53.89915 \\
\hline
\end{tabular}

\section{Conclusion}

In this paper, the problem of pricing over the counter (OTC) CAT risk bond contracts was investigated between an insurance company and a hedge-fund. This is of great importance for both the insurance company and the hedge-fund, as CAT risk bonds are always part of a multi-asset class portfolio of alternative premia in many hedge-funds, (see CPA (2015)). The method that we use is formulated based on the indifference utility pricing method and the derived framework and analysis is from the insurance company point of view. Under those assumptions no dynamic trading is involved. Furthermore, a risk neutral and a risk averse cases are investigated. Finally, as an illustration of the theoretical findings, a CAT risk bond related to agriculture catastrophes (temperature) designed analytical for two major cities in Iran. Thus, the demand curves for CAT bonds were computed and portfolio of CAT bonds was constructed. The numerical findings are demonstrated in numerous tables and figures. As a future extension of the present paper, more catastrophe risk parameters as well as stochastic interest rate will be considered. In addition, one can consider pricing the insurance contract within this framework when one needs to balance the market demand against the CAT bond demand function.

\section{Acknowledgements}

The authors would like to acknowledge the gracious support of this work through the EPSRC and ESRC Centre for Doctoral Training on Quantification and Management of Risk and Uncertainty in Complex Systems and Environment (EP/L015927/1). This paper has been benefited from comments of participants at the 1st Symposium in Quantitative Finance and Risk Analysis in 2015, in Santorini, Greece, at the following seminars: Department of Mathematical Sciences (University of Liverpool), Management School (University of Liverpool), Department of Economics (Concordia University). We would also like to thank the anonymous reviewers for the helpful comments.

\section{References}

Arrow, K.J., The theory of risk aversion. Essays in the theory of risk-bearing, 1971, pp. 90-120.

Baryshnikov, Y., Mayo, A. and Taylor, D., Pricing of CAT bonds. preprint, 2001.

Bilal, A., Pantelous, A.A. and Shao, J., Towards resilience to nuclear accidents: financing nuclear liabilities via catastrophe risk bonds. ASCE-ASME Journal of Risk and Uncertainty in Engieering Systems: Part B., 2015, pp. 1-16. 
Braun, A., Determinants of the cat bond spread at issuance. Zeitschrift für die gesamte Versicherungswissenschaft, 2012, 101, 721-736.

Burnecki, K. and Kukla, G., Pricing of zero-coupon and coupon CAT bonds. Applicationes Mathematicae, 2003, 30, 315-324.

Burnecki, K., Kukla, G. and Taylor, D., Pricing of catastrophe bonds. In Statistical Tools for Finance and Insurance, pp. 371-391, 2011, Springer.

Carmona, R., Indifference pricing: theory and applications, 2009, Princeton University Press.

Carr, P., A Note on the Pricing of Commodity-Linked Bonds. The Journal of Finance, 1987, 42, 1071-1076.

Cox, J.C. and Schwartz, E.S., The Pricing of Commodity-Linked Bonds. The journal of Finance, 1982, 37, 525-539.

Cox, S.H., Fairchild, J.R. and Pedersen, H., Economic aspects of securitization of risk. ASTIN Bulletin, 2000, 30, 157-193.

Cox, S.H. and Pedersen, H.W., Catastrophe risk bonds. North American Actuarial Journal, 2000, 4, 56-82.

CPA, Clear Path Analysis: Inusrance-linked securities for institutional investors. Report, 2015, pp. 1-53.

Cummins, J.D., CAT Bonds and Other Risk-Linked Securities: State of the Market and Recent Developments. Risk Management and Insurance Review, 2008, 11, 23-47.

Cummins, J.D. and Mahul, O., Catastrophe risk financing in developing countries: principles for public intervention, 2009, World Bank Publications.

Dieckmann, S., A consumption-based evaluation of the cat bond market. Technical report, Working paper, University of Pennsylvania, 2011.

Duncan, J. and Myers, R.J., Crop insurance under catastrophic risk. American Journal of Agricultural Economics, 2000, 82, 842-855.

Egami, M. and Young, V.R., Indifference prices of structured catastrophe (CAT) bonds. Insurance: Mathematics and Economics, 2008, 42, 771-778.

Föllmer, H. and Schweizer, M., Hedging of contingent claims under incomplete information. Applied Stochastic Analysis, 1991, 5, 389-414.

Froot, K.A. and Posner, S., Issues in the pricing of catastrophe risk. Trade Notes, Marsh E McLennan Securities, 2000.

Froot, K.A. and Posner, S.E., The pricing of event risks with parameter uncertainty. The Geneva Papers on Risk and Insurance Theory, 2002, 27, 153-165.

Galeotti, M., Gürtler, M. and Winkelvos, C., Accuracy of Premium Calculation Models for CAT Bonds-An Empirical Analysis. Available at SSRN 1392734, 2011.

GAO, Catastrophe insurance risk: The Role of Risk-Linked Securities and Factors Affecting Their Use, 2002, Gao-02-941.

Gerber, H.U. and Pafum, G., Utility functions: from risk theory to finance. North American Actuarial Journal, 1998, 2, 74-91.

Gibson, R. and Schwartz, E.S., Stochastic convenience yield and the pricing of oil contingent claims. The Journal of Finance, 1990, 45, 959-976.

Hagedorn, D., Heigl, C., Mueller, A. and Seidler, G., Choice of triggers. The Handbook of Insurance-Linked Securities,, 2009, pp. 37-48.

Härdle, W.K. and Cabrera, B.L., Calibrating CAT bonds for Mexican earthquakes. Journal of Risk and Insurance, 2010, 77, 625-650.

Harrison, M.J. and Kreps, D.M., Martingales and arbitrage in multiperiod securities markets. Journal of Economic Theory, 1979, 20, 381-408.

Jarrow, R.A., A simple robust model for CAT bond valuation. Finance Research Letters, 2010, 7, 72-79.

Lee, J.P. and Yu, M.T., Pricing default-risky CAT bonds with moral hazard and basis risk. Journal of Risk and Insurance, 2002, 69, 25-44.

Lee, J.P. and Yu, M.T., Valuation of catastrophe reinsurance with catastrophe bonds. Insurance: Mathematics and Economics, 2007, 41, 264-278.

Lin, S.K., Shyu, D. and Chang, C.C., Pricing catastrophe insurance products in Markov jump diffusion models. Journal of Financial Studies, 2008, 16, 1-34.

Lin, Y. and Cox, S.H., Securitization of mortality risks in life annuities. Journal of Risk and Insurance, 2005, 72, 227-252.

Lin, Y. and Cox, S.H., Securitization of catastrophe mortality risks. Insurance: Mathematics and Economics, 2008, 42, 628-637.

Ma, Z.G. and Ma, C.Q., Pricing catastrophe risk bonds: A mixed approximation method. Insurance: Math- 
ematics and Economics, 2013, 52, 243-254.

Miltersen, K.R. and Schwartz, E.S., Pricing of options on commodity futures with stochastic term structures of convenience yields and interest rates. Journal of Financial and Quantitative Analysis, 1998, 33, 3359.

Miranda, M. and Vedenov, D.V., Innovations in agricultural and natural disaster insurance. American Journal of Agricultural Economics, 2001, pp. 650-655.

Monoyios, M., Utility indifference pricing with market incompleteness. Nonlinear Models in Mathematical Finance: New Research Trends in Option Pricing, 2008, pp. 67-100.

Nowak, P. and Romaniuk, M., Pricing and simulations of catastrophe bonds. Insurance: Mathematics and Economics, 2013, 52, 18-28.

O'Hara, M., Commodity bonds and consumption risks. The Journal of Finance, 1984, 39, 193-206.

Pantelous, A.A. and Passalidou, E., Optimal quadratic control of a nonlinear premium-reserve model in a competitive insurance market. Preprint, 2015.

Pelsser, A., On the applicability of the Wang transform for pricing financial risks. ASTIN Bulletin, 2008, 38, 171.

Pérez-Fructuoso, M.J., Modeling loss index triggers for CAT bonds: a continuous approach. Variance, 2008, 2, 253-265.

Reshetar, G., Pricing of multiple-event coupon paying CAT bond. Available at SSRN 1059021, 2008.

Schwartz, E.S., The stochastic behavior of commodity prices: Implications for valuation and hedging. The Journal of Finance, 1997, 52, 923-973.

Schweizer, M., On the minimal martingale measure and the möllmer-schweizer decomposition. Stochastic Analysis and Applications, 1995, 13, 573-599.

Shao, J., Modelling Catastrophe Risk Bonds, 2015, PhD Thesis, University of Liverpool, Liverpool United Kingdom.

Shao, J., Pantelous, A.A. and Papaioannou, A., Catastrophe risk bonds with applications to earthquakes. European Actuarial Journal, 2015a, pp. 1-26.

Shao, J., Papaioannou, A. and Pantelous, A.A., Pricing and simulating CAT risk bonds in a Markovdependent environment. Preprint, 2015b.

Skees, J.R. and Barnett, B.J., Conceptual and practical considerations for sharing catastrophic/systemic risks. Review of Agricultural Economics, 1999, 21, 424-441.

Skees, J.R. and Barnett, B.J., Enhancing microfinance using index-based risk-transfer products. Agricultural Finance Review, 2006, 66, 235-250.

Snyder, R.L. and Melo-Abreu, J.P., Frost protection: fundamentals, practice and economics. Volume 1, 2005, FAO.

Sun, L., Turvey, C.G. and Jarrow, R.A., Designing catastrophic bonds for catastrophic risks in agriculture. Agricultural Finance Review, 2015.

Turvey, C.G., Managing food industry business and financial risks with commodity-linked credit instruments. Agribusiness, 2006, 22, 523-545.

Turvey, C.G., The pricing, structure, and function of weather-linked bonds, mortgages, and operating credit. Agricultural Finance Review, 2008, 68, 135-150.

Vaugirard, V.E., Pricing catastrophe bonds by an arbitrage approach. The Quarterly Review of Economics and Finance, 2003, 43, 119-132.

Vedenov, D.V., Epperson, J.E. and Barnett, B.J., Designing catastrophe bonds to securitize systemic risks in agriculture: the case of Georgia cotton. Journal of Agricultural and Resource Economics, 2006, pp. 318-338.

Wakker, P.P., Explaining the characteristics of the power (CRRA) utility family. Health Economics, 2008, 17, 1329-1344.

Wang, S.S., Cat bond pricing using probability transforms. Geneva Papers: Etudes et Dossiers, special issue on Insurance and the State of the Art in Cat Bond Pricing, 2004, 278, 19-29.

Young, V.R., Pricing in an incomplete market with an affine term structure. Mathematical Finance, 2004, 14, 359-381.

Zhu, W., Ambiguity aversion and an intertemporal equilibrium model of catastrophe-linked securities pricing. Insurance: Mathematics and Economics, 2011, 49, 38-46.

Zimbidis, A.A., Frangos, N.E. and Pantelous, A.A., Modeling earthquake risk via extreme value theory and pricing the respective catastrophe bonds. ASTIN Bulletin, 2007, 37, 163-184. 